LIE GUO, Ph.D.

(Corresponding author)

E-mail: guo_lie@dlut.edu.cn

MINGHENG ZHANG, Ph.D.

E-mail: gloriazhang@163.com

LINHUI LI, Associate Professor, Ph.D.

E-mail: 40301213@qq.com

YIBING ZHAO, Ph.D.

E-mail: zhaoyibing005@163.com

School of Automotive Engineering, Dalian University of

Technology

No.2 Linggong Road, Ganjingzi District, Dalian 116024,

China

YINGZI LIN, Ph.D.

E-mail: yi.lin@neu.edu

Department of Mechanical and Industrial Engineering

College of Engineering, Northeastern University

360 Huntington Avenue, Boston, MA 02115, USA
Traffic Engineering

Preliminary Communication

Submitted: Jan. 12, 2015

Accepted: Dec. 1, 2015

\title{
BODY PARTS FEATURES-BASED PEDESTRIAN DETECTION FOR ACTIVE PEDESTRIAN PROTECTION SYSTEM
}

\begin{abstract}
A novel pedestrian detection system based on vision in urban traffic situations is presented to help the driver perceive the pedestrian ahead of the vehicle. To enhance the accuracy and to decrease the time spent on pedestrian detection in such complicated situations, the pedestrian is detected by dividing their body into several parts according to their corresponding features in the image. The candidate pedestrian leg is segmented based on the gentle AdaBoost algorithm by training the optimized histogram of gradient features. The candidate pedestrian head is located by matching the pedestrian head and shoulder model above the region of the candidate leg. Then the candidate leg, head and shoulder are combined by parts constraint and threshold adjustment to verify the existence of the pedestrian. Final$l y$, the experiments in real urban traffic circumstances were conducted. The results show that the proposed pedestrian detection method can achieve pedestrian detection rate of $92.1 \%$ with the average detection time of $0.2257 \mathrm{~s}$.
\end{abstract}

\section{KEY WORDS}

automobile safety; pedestrian protection; gentle AdaBoost; template matching;

\section{INTRODUCTION}

With the increase of automobile ownership and rapid development of transportation infrastructures, numerous traffic accidents have done great damage to human lives and properties, as well as to national economies every year all over the world. Therefore, the necessity to avoid traffic accidents, as well as improve automobile safety, has aroused much concern by governments and social organizations. Among these traffic accidents, pedestrians are the most vulnerable road users and are more likely to suffer from those accidents under urban transportation environments. The crashes between vehicle and pedestrian kill many lives each year. The World Health Organization (WHO) has found that almost half of the estimated 1.24 million people who get killed in road traffic crashes every year are pedestrians (22\%), motorcyclists (23\%) and cyclists (5\%) [1]. According to the statistics from the National Highway Traffic Safety Administration (NHT$\mathrm{SA})$, an estimated $5,615,000$ police-reported crashes in 2012 resulted in 4,743 pedestrians killed and 76,000 pedestrians injured which accounts for $14.1 \%$ and $3.2 \%$, respectively [2].

As for China, the traffic situation is not satisfactory. China is a typical mixed traffic dominated country with complex and diversiform traffic road circumstance. It is common to see street scene of China with both pedestrians and vehicles, which worsens the problem of pedestrian safety in China. The rapid rate of motorization taking place in China means that road traffic crashes and associated non-fatal and fatal injuries will soon become a bigger societal and public safety problem than before in the absence of appropriate road traffic crash prevention measures [3]. Currently, the pedestrians have ranked second to drivers as priority population requiring protection. According to the report of the China Ministry of Public Security, there were 219,521 road traffic accidents in 2010, resulting in 16,281 pedestrians killed and 44,629 pedestrians injured, which accounts for $25.0 \%$ and $17.6 \%$, respectively [4].

Fortunately, many countries have carried out their laws and regulations to protect pedestrians and other 
vulnerable road users [5]. In order to protect vulnerable pedestrians' safety effectively, automobile safety should be improved accompanied by drafting and implementing laws in relation to these problems. Automobile safety technology consists of active safety and passive safety technology [6]. Automobile passive safety technology aims at preventing or minimizing injury when a crash is unavoidable. The automobile active safety technology is helpful to reduce the chance of a crash by providing the driver with better means of controlling the automobile and avoiding hazards. Šimunović [7] indicated that it is important to integrate the pedestrian traffic with the future of intelligent transport system architecture on time.

With an aim trying to protect pedestrians from being hurt and avoiding great damage when the crashes happen, studies of automobile passive safety technologies mainly concentrate on pedestrian-friendly vehicle design, collision bumper, biomechanics, crash reconstruction analysis, and so on. For example, Kopczynski et al. [8] validated the design of the frontal pedestrian protection system. Matsui et al. [9] studied the performance of the collision damage mitigation braking system to evaluate the effect of reducing impact velocity on mitigating pedestrian injury. The goal of automobile active safety designs for pedestrian protection is to avoid a pedestrian crash or reduce the impact speed based on the pedestrian detecting results [10]. Broggi et al. [11] presented a pedestrian protection system based on a scenario-driven search method to locate potentially dangerous situations by considering the information coming from vision and laser sensors. Rosén et al. [12] indicated that the effectiveness of reducing fatally injured pedestrians in frontal collisions with cars reached $40 \%$ at a field of view of $40^{\circ}$ by introducing the autonomous brakes. Keller et al. [13] presented an active pedestrian safety system by detecting the pedestrian based on stereo vision and control the vehicle by automatic braking and evasive steering. Studies show that effective active crash avoidance measures combining perfect active safety system applied to automobile can provide greater possibility of implementing additional passive deployable features to ensure better protection for pedestrians, such as bumper airbag and hood leading edge airbag. However, the reliability and effectiveness of such active sensing system should be enhanced.

This paper presents a novel pedestrian detection system based on vision to realize active pedestrian protection in urban traffic situations. Currently, the key technology of pedestrian detection is to find out discriminative features to distinguish pedestrians from the background. Previous studies concentrated on utilizing local filters to select the most appropriate feature subset from pixel intensities, such as Haar wavelet features [14]. Recent studies have demonstrated the superiority of adaptive local pixel or edge field features over non-adaptive Haar wavelet features when identifying a pedestrian. For example, Lowe [15] presented the selected image region with an associated descriptor named scale invariant feature transform (SIFT) to recognize a pedestrian with clutter and occlusion. Inspired by SIFT, Dalal and Triggs [16] introduced histograms of oriented gradient (HOG) features, which are particularly suited for human detection in images. HOG and variant of HOG features have gained much concern because of their efficiency, such as PCA-HOG [17] and HOG-LBP [18] features. In 2012, Armanfard and Komeili [19] announced that collect local binary pattern (LBP) in vertical edges can do better in detecting pedestrians as they found that pedestrian tends to show distinctive vertical edges in an image. Though these features show good recognition performance to certain pedestrian datasets, they are time-costing because of the high dimensionality of the feature vectors, which is not suitable to real-time automobile safety system. Actually, urban traffic situations are complex and the pedestrians are diverse and random, and using one type of feature subset to realize overall pedestrian is challenging. Therefore, many scholars showed their detecting methods using information fusion. For example, Zhang et al. [20] trained the classifiers by SVM and AdaBoost combined HOG and Edgelet features to detect pedestrians and the detection of pedestrians in infrared images has proven to perform well. Sun et al. [21] mixed the simplified HOG and LBP features for pedestrian detection.

Studies show that the multi-feature fusion can do a lot to increase the pedestrian detection precision. But further research still needs to be done to determine which feature is appropriate for the detection of the human body and its parts, such as the leg and the head. Still, there is a lot of work which needs to be done to deal with the classification accuracy and the detecting speed by designing the composite structure of classifier to make full use of each feature or each body part [22]. To utilize the appropriate features of each body part and to enhance the detection precision, this paper presents a body part based pedestrian detection.

The remainder of this paper is arranged as follows. Section 2 briefly introduces the body part based pedestrian detection procedure utilizing the look-up table gentle AdaBoost to train the optimized HOG features and template matching method. Experiments are presented in Section 3. Section 4 concludes the paper and gives some future works.

\section{METHODOLOGY}

\subsection{Leg detection based on optimized HOG features}

Current studies mainly concentrate on the full body detection utilizing discriminative features, such as HOG, HOG-LBP and Haar-like features. In Dalal's work 
[16], each detection window is divided into cells of $8 \times 8$ pixels and each group of $2 \times 2$ cells are integrated into a block. Descriptors in blocks are represented by a 36D feature vector. Each $64 \times 128$ detection window is represented by $7 \times 15$ blocks, giving a total of 3,780 dimensional feature vectors per detection window. Obviously, the computation is large either in the feature extraction or classifier training.

Actually, employing different feature extraction methods according to the visual characteristics of different body parts will get more remarkable detection effects, especially for the pedestrian legs. When legs occupy the most part of the image, the edges are more obvious and histograms will show a maximum value in certain gradient orientation. A peak value will appear in certain gradient directions and gradient amplitude, while the background of the region where the legs exist is usually road surface image. In this paper, the optimized HOG features are applied to segment the pedestrian's legs, which is calculated in the bottom half of the detection window $(64 \times 64)$. Following the same size of cells and blocks of Dalal's [16], the dimension of the feature vector is reduced to $1,386 \mathrm{D}$.

Theoretically speaking, every feature can be a reflection of the target's contour information to some extent. And the more characteristic dimensions there are, the more precise is the description of the target. However, significant reduction in dimension of the feature vector improves the training and testing efficiency. Actually, only a small part of all of the features is conclusive for the target classification. The redundancy features will make the algorithm more complex and prolong the time for classifier training and detecting. Therefore, after abstracting the HOG features, the dimensionalities are reduced by utilizing the weighted linear discriminant analysis (WLDA) method [23] WLDA projects the multidimensional characteristics of the samples to a one-dimensional straight line and separates different kinds of samples by changing the direction of projection. The process of this projection is functional in weak classifier and is able to reduce its dimension. The optimal projecting direction can be determined by [24]

$$
\left\{\begin{array}{l}
\bar{m}=\frac{1}{n \sum_{i=1}^{n} w_{i}} \sum_{i=1}^{n} w_{i} f\left(x_{i}\right) \\
\bar{S}=\frac{1}{(n-1) \sum_{i=1}^{n} w_{i}^{2}} \sum_{i=1}^{n} w_{i}^{2}\left(f\left(x_{i}\right)-\bar{m}\right)\left(f_{i}\left(x_{i}\right)-\bar{m}\right)^{T} \\
a^{*}=\left(\bar{S}^{(1)}+\bar{S}^{(2)}\right)^{-1}\left(\bar{m}^{(1)}-\bar{m}^{(2)}\right)
\end{array}\right.
$$

where $w_{i}$ represents the weight of sample $i, f\left(x_{i}\right)$ is the vector feature for sample $i, n$ is the number of the sample, $\bar{m}^{(1)}$ and $\bar{m}^{(2)}$ are the intra-class mean values of pedestrian and non-pedestrian samples respectively, $\bar{S}^{(1)}$ and $\bar{S}^{(2)}$ are the intra-class weighted covariance matrices of pedestrian and non-pedestrian samples, respectively, $a^{*}$ is the best weighted projecting direction.

To enhance the expressive abilities of the weak classifiers, gentle AdaBoost algorithm is adopted to train those optimized HOG features. The gentle AdaBoost algorithm is a variant of the powerful boosting learning technique, which has a higher detection rate and a lower false positive rate than the basic discrete AdaBoost [25]. The output function of each weak classifier can be given as

$h_{1}(x)=P_{w}(y=+1 \mid x)-P_{w}(y=-1 \mid x)$

where $h_{t}(x)$ is the weak classifier, $P_{w}(y=1 \mid x)$ and $P_{w}(y=-1 \mid x)$ are the weighted probabilities when the sample with feature vector $x$ belongs to the pedestrian and non-pedestrian samples.

Then the final classifier can be defined as

$H_{T}(x)=\operatorname{sign}\left[\sum_{t=1}^{T} h_{t}(x)\right]$

where $T$ is the number of training times and $H_{T}(x)$ is the final strong classifier for pedestrian leg detection.

To train the classifier utilizing the gentle AdaBoost algorithm, a look-up table (LUT) algorithm is adopted to estimate the posterior probability. According to the principle of LUT algorithm, the feature values of the sample are divided into a certain number of non-intersected subsets. For a sample with eigenvalue $x$, the output of the weak classifier is the difference between the weighted probabilities of pedestrian and non-pedestrian samples. Obviously, the output of LUT weak classifier is a real value representing confidence coefficient which is more adaptive to the distribution of samples and helps to enhance the classifying precision [26].

After performing the weighted linear discriminant analysis, the HOG features are projected into one dimension. They are normalized to fall within the range of $[0,1]$ and then they are divided into the number of k subintervals equally

$\operatorname{bin}_{j}=[(j-1) / k, j / k], \quad j=1,2, \ldots, k$

The weak classifier can be defined as

if $f(x) \in \operatorname{bin}_{j}$ then $h(x)=W_{j}^{(1)}-W_{j}^{(2)}$

where $W_{j}^{(1)}$ and $W_{j}^{(2)}$ are the conditional probability for the feature value of pedestrian and non-pedestrian samples that fall in the range of bin $_{i}$ respectively, they can be achieved by

$$
\left\{\begin{array}{l}
W_{j}^{(1)}=P_{w}\left(f(x) \in \operatorname{bin}_{j}, y=+1\right), \quad j=1,2,, k \\
W^{(2)}=P_{w}(f(x) \in \operatorname{bin}, y=-1), \quad j=1,2, k
\end{array}\right.
$$

To be specific, set $D_{j}^{(1)}$ and $D_{j}^{(2)}$ to be the sum of the weight value the pedestrian and non-pedestrian samples that fall in the interval of bin $_{i}$ respectively 


$$
\left\{\begin{array}{l}
D_{j}^{(1)}=\sum_{f\left(x_{i}\right) \in \operatorname{bin}_{j} \delta y_{i}=1} W_{i} \\
D_{j}^{(2)}=\sum_{f\left(x_{i}\right) \in b_{i n} j \& y_{i}=-1} W_{i}
\end{array}\right.
$$

Then the conditional probabilities are

$$
\left\{\begin{array}{l}
P_{\mathrm{w}}\left(f(x) \in \operatorname{bin}_{j}, y=1\right)=\frac{D_{j}^{(1)}}{D_{j}^{(1)}+D_{j}^{(2)}} \\
P_{\mathrm{w}}\left(f(x) \in \operatorname{bin}_{j}, y=-1\right)=\frac{D_{j}^{(2)}}{D_{j}^{(1)}+D_{j}^{(2)}}
\end{array}\right.
$$

Define

$$
\operatorname{Bin}_{k}^{j}(u)=\left\{\begin{array}{l}
1 u \in[j-1 / k, j / k) \\
0 u \notin[j-1 / k, j / k)
\end{array} j=1,2, k\right.
$$

Then the weak classifier based on LUT gentle AdaBoost can be defined as

$$
h_{\text {LUT }}(x)=\frac{D_{j}^{(1)}-D_{j}^{(2)}}{D_{j}^{(1)}+D_{j}^{(2)}} \operatorname{Bin}_{k}^{j}(f(x))
$$

After defining the weak classifier, the gentle AdaBoost algorithm is utilized to train the final strong classifier from the weak classifier pool. The strong classifier is a linear combination of a series of weak classifiers selected, as shown in (3). Training is stopped when the false alarm rate on the training set reaches a given threshold.

\subsection{Head and shoulder detection based on template matching}

As a non-rigid target, the human body will show different gestures, which makes it hard to describe a pedestrian by a uniform model. It has been found that the variability of head contour above the shoulder tends to be very small no matter whether it is a front view or a side view. Some different kinds of template can be defined to adapt to the need of matching as the head has a certain shape in the image. Besides, the head is not easy to be occluded and can be extracted easily. This means that the template matching is very appropriate to head detection. This paper locates the head and shoulder by matching an " $\Omega$ " bitmap map above the assigned area of the detected legs, as shown in Figure 1 . The size of the head and shoulder contour template is $32 \times 28$ for a $64 \times 128$ pedestrian sample. The template is a binary array which takes on the value 1 at a silhouette edge and 0 elsewhere.

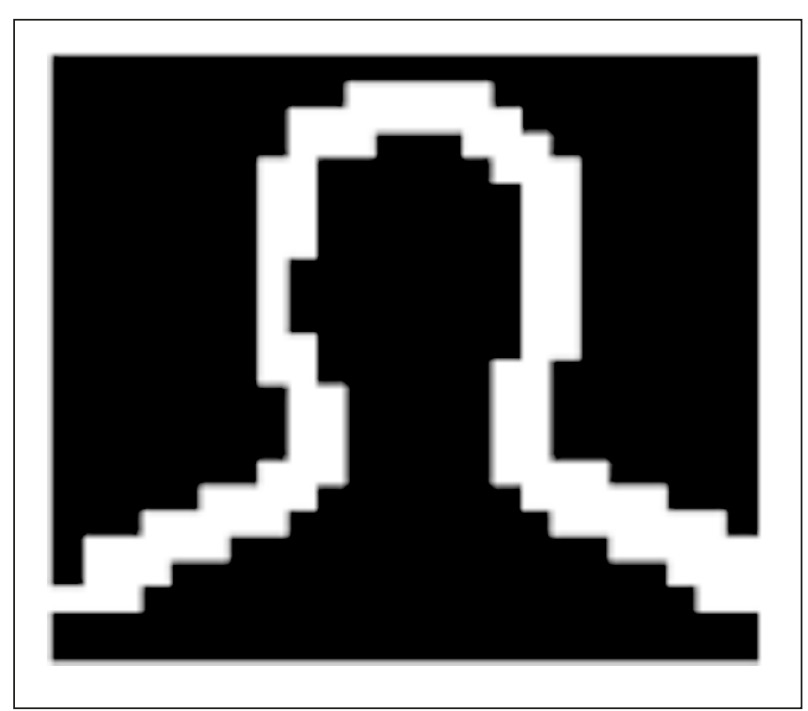

Figure 1 - Head and shoulder contour template

Generally, template matching is realized in distance transform (DT) images. For example, Gavrila and Munder [27] converted the candidate windows to be matched in DT images during the template matching procedure. In this way the distance measure can be presented by smooth function of template transform parameters. The benefit of matching a template with the DT image rather than with the edge image is that the similarity measure will be smoother as a function of the template transformation parameters [28]. The benefit thereof is that it allows some degree of dissimilarity between the template and the object of interest in the image, which helps to improve the matching precision. Matching a template and an image involves computing the edge of the image and applying a distance transform to obtain a DT image. Figure 2 shows an example of edge image and its corresponding DT image.

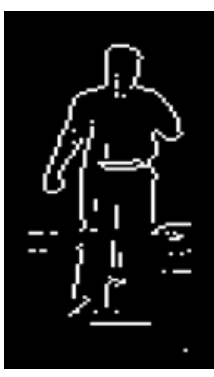

a) Edge image

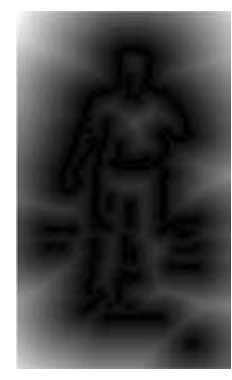

b) DT image
Figure 2 - Result of distance transform

After computing the edges of the image and conducting the distance transform, the matching process is begun by the convolution transforming the head and 
shoulder template with the DT image. Chamfer matching is a special case of template matching [29]. The template $\mathrm{F}$ is shifted over an edge-mapped image DT and for each position the distance metric $D_{\text {chamfer }}$ is computed, which is defined as

$D_{\text {chamfer }}(F, D T)=\frac{1}{F} \sum_{f \in F} D T(f)$

where $D T(f)$ is the Euclid distance of $f$ and corresponds to DT image.

The more similar the images are, the smaller is the Chamfer distance. To an image waiting for matching, the Chamfer distance of the region without head is greater than the region including head. The principle for choosing the template-matching principle is that the region with minimum Chamfer distance is taken as the size and position of head optimum box.

To improve the template-matching precision, the matching regions are constricted by the constraints between different human body parts. Commonly, the head will not appear at the bottom or the sides of this region. Additionally, the detection of legs can be the reference for the detection of head. According to the definition of human body parts [30], the scope of template matching is limited to a region of $0.3 \mathrm{~h} \times 0.75 \mathrm{w}$ rectangular area above the region where the legs are detected, as shown in Figure 3.

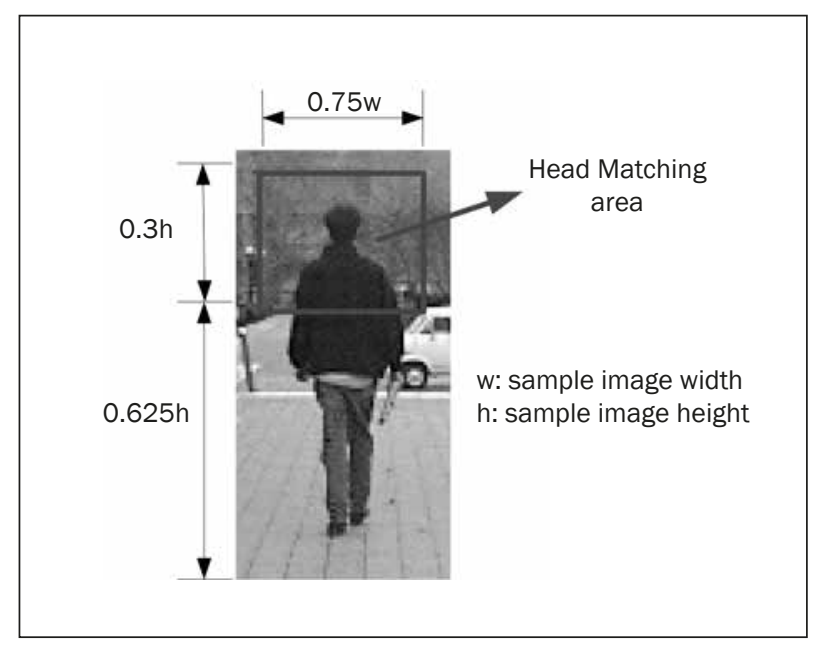

Figure 3 - Illustration of head matching area on a pedestrian sample

As shown in Figure 3, the pedestrian head can be located using the above template-matching method after the detection of pedestrian legs in the image.

\subsection{Pedestrian detection by combining body parts detection results}

The performance of classifiers can be affected by the complex edge gradients of the background which will decrease the precision of the algorithm in real world [31]. Of all the methods, the way based on multi-feature fusion can adapt to many scenes and has a higher detection rate than others. Traditional methods of feature fusion merge together different features to detect the same target. For example, Grassi et al. [32] combined different invariant features from a lidar sensor and an infrared camera to detect the pedestrian, while in this paper, different parts of the pedestrian with various features are combined to improve the pedestrian recognition ability.

In the process of pedestrian detection, the target's legs are first positioned to have an initial position of the pedestrian. Then, a region which is similar to the pedestrian's head will be located using the template-matching method in the specific area shown in Figure 3. The form features are transformed to numerical characteristics after analysing the difference between pedestrian's head and the background. Then a classifier combined with support vector machine (SVM) is trained for head detection. Additionally, some false alarms can be avoided if the matching regions are restricted by body parts' correlation constraints. Finally, the fusion of the above detecting results can judge whether the target is a pedestrian or not.

A region resembling the contour of pedestrian head is segmented after template matching. However, it is difficult to determine whether this region is a pedestrian or not by judging the Chamfer distance with a single threshold. Therefore, this kind of head feature can be converted to a numerical value that can be taken as an auxiliary judgment standard, such as circular similarity and moment of edges [33]. Then we can combine the results of head detection with the leg detection. The ideal situation for the determination of the candidate is that the decision is in agreement with that of legs. Then the target can be seen as pedestrian definitively. Actually, the determination results of head and leg detection may not match each other sometimes. Therefore, a classification threshold should be adjusted to fuse the classification results of head and leg detection, as shown in Figure 4.

According to the schematic of classification threshold adjustment, if the threshold is decreased, the feature point located in non-pedestrian side when the threshold is zero will be judged to be a pedestrian. On the contrary, the feature point located in pedestrian side when the threshold is zero will be judged to be a non-pedestrian if the threshold is increased, which means that the classification is very rigorous and the candidate pedestrian is likely to be taken as a non-pedestrian. The main purpose of the head detection is to avoid false alarm on the detection of legs. Therefore, the threshold adjustment should be based on the existence of head profile features. If no head is detected in head classifiers, rigorous threshold adjustment should be carried out to increase the decision value of legs classifiers and then another determination on legs should be done. The decision values of the legs 

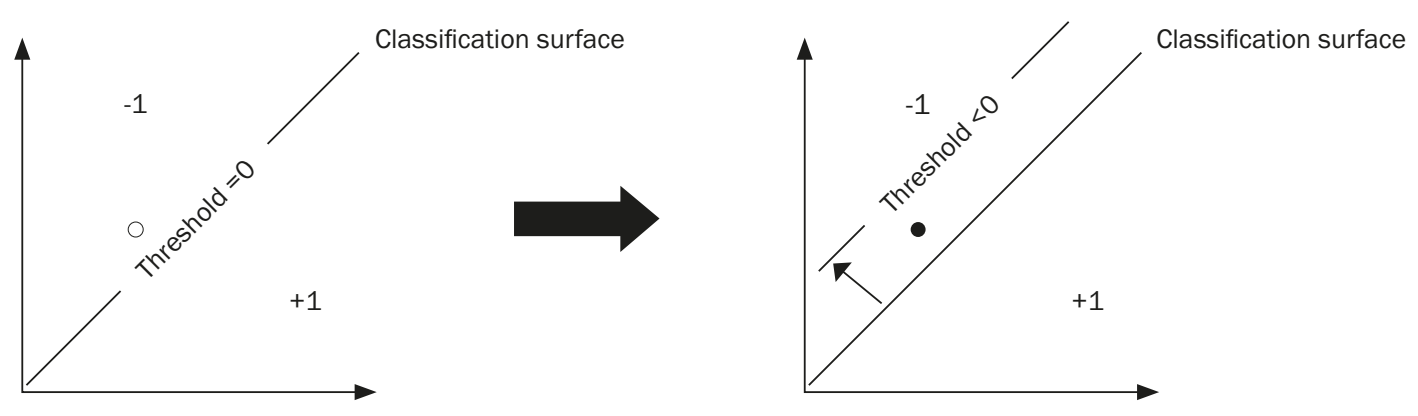

a) Decrease classification threshold
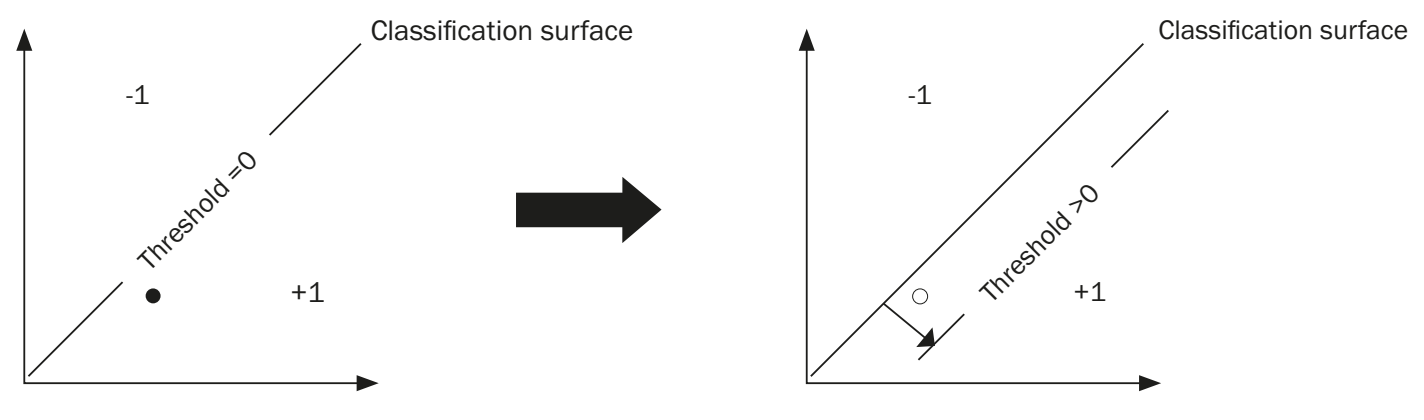

b) Increase classification threshold

Figure 4 - The schematic diagram of threshold adjustment

and head classifiers are defined as $V_{1}$ and $V_{2}$, respectively. $V_{1}^{\prime}$ and $V_{2}^{\prime}$ are the corresponding decision values after threshold adjustment. $T_{1}$ and $T_{2}$ is the classification threshold of leg and head, respectively; they are initialized to zero; $\alpha$ is the sum of the weighted leg weak classifiers. The steps for the adjustment interval of the legs threshold and head threshold are $S_{1}$ and $S_{2}$, which are set to be 2 and 0.5 , respectively. The detailed adjustment procedure for body parts combination is shown in Figure 5.

During the pedestrian detection procedure, the pedestrian legs are first detected based on the optimized HOG features and outputs the decision value $V_{1}$. Then the pedestrian head is further located based on template-matching method and outputs the decision value $V_{2}$. The threshold is adjusted according to these decision values, as shown in Figure 5, to determine whether or not the image is a pedestrian.

\section{EXPERIMENT AND ANALYSIS}

Simulation experiments are performed for the proposed pedestrian detection system. The program is coded using $\mathrm{VC}++$. The system runs on a PC with an Intel Dual Core 2.66GHz processor with $2 \mathrm{~GB}$ of RAM. The camera type is Stingray F033 from AVT, installed on the intelligent vehicle prototype developed by our research group. The image from the camera, whose size is $640 \times 480$ pixels, is scaled to $320 \times 240$ pixels to detect the pedestrian. The detailed architecture of the intelligent vehicle system is presented in our previous work [34].

The training and testing pedestrian samples are selected from the INRIA pedestrian dataset and a dataset of images in urban traffic environment collected by our research group. The training samples consist of 950 pedestrian samples and 1,020 non-pedestrian samples, which are used to train the leg detection classifier. The testing samples consist of 890 pedestrian samples and 906 non-pedestrian samples, which are used to validate the leg detection classifier. The lower half parts of all sample images were scaled to $64 \times 64$ pixels, in which the legs appear mostly.

Unlike the traditional HOG based pedestrian method utilizing linear SVM to train the samples, the WLDA is adopted to reduce the dimension of HOG features and the conventional threshold weak classifiers are replaced by LUT weak classifiers. The number of weak classifiers trained by the LUK gentle AdaBoost is 28 , after utilizing the WLDA to optimize the HOG features. A set of weak classifiers were selected to compose the final strong classifier to detect the pedestrian leg. To validate the performance of the leg detection based on optimized HOG features and LUT gentle AdaBoost, three different types of weak classifiers were tested 


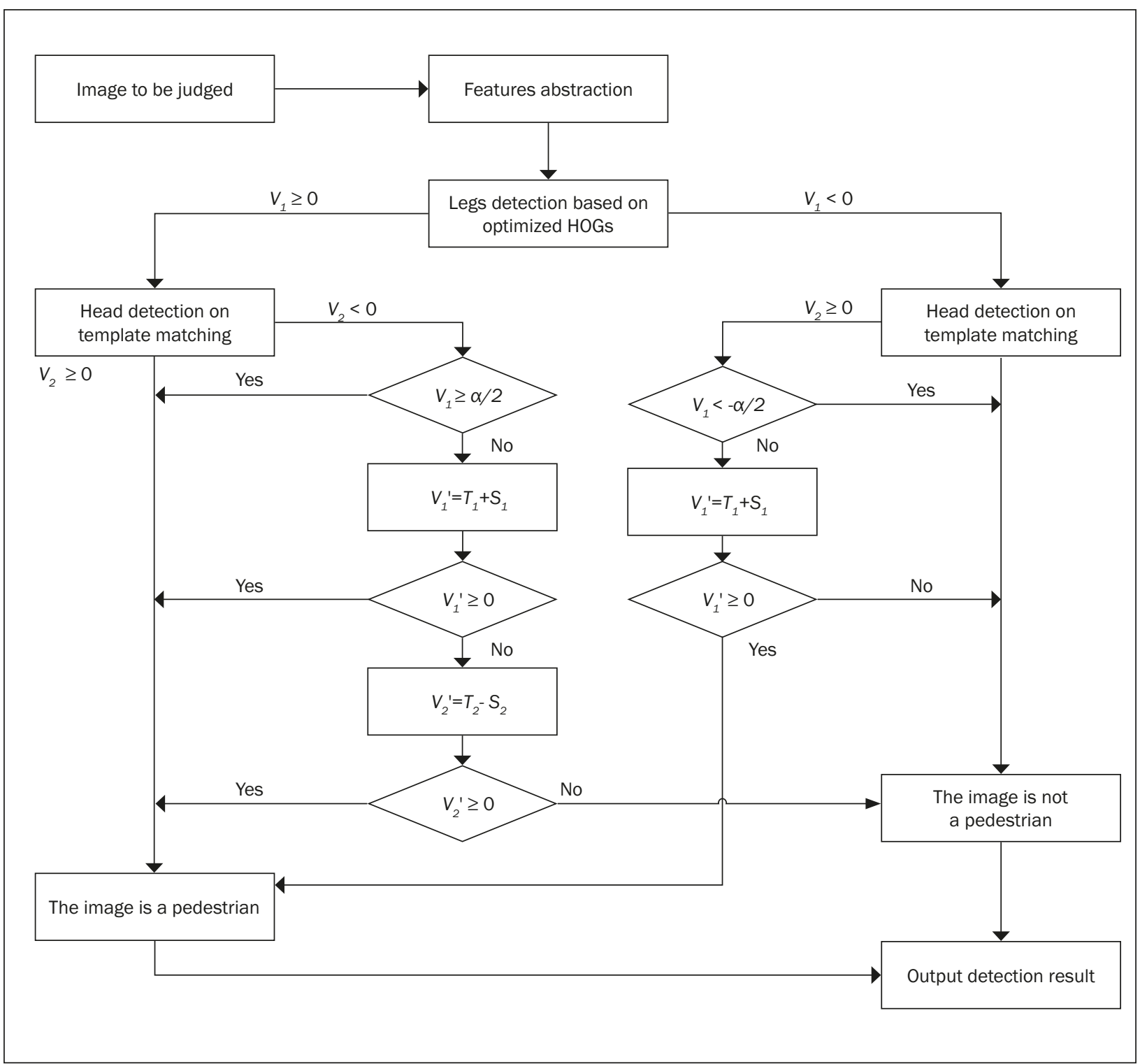

Figure 5 - Classification adjustment diagram for body parts combination

on the tested pedestrian samples. They are the linear SVM, the WLDA with fixed threshold, and the WLDA with LUT gentle AdaBoost. The leg detection results on the testing samples are compared using the ROC curve, as shown in Figure 6. Table 1 compares the time consumption and the detection performance.

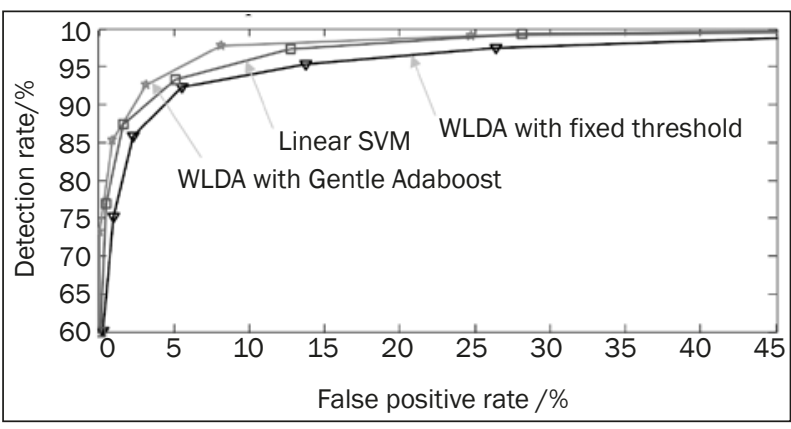

Figure 6 - ROC curves for leg detection on testing samples
The ROC curve in Figure 6 shows that the accuracy of AdaBoost detector is improved by using LUT weak classifiers rather than fixed threshold weak classifiers. As to the time consumption, the two kinds of WLDA weak classifiers are reduced by $67.4 \%$ and $64.5 \%$, respectively compared with linear SVM proving the great superiority in operation speed. Figure 7 displays several detection results of the pedestrian's leg.

The validity of pedestrian detection based on body parts combination were tested on the testing samples; the results are shown in Table 2. A total of 890 pedestrian and 906 non-pedestrian samples were tested. The detection rate was $92.1 \%$ for the pedestrian samples and $95.0 \%$ for the non-pedestrian samples.

The proposed pedestrian detection method was conducted on the intelligent vehicle prototype to verify its performance in real traffic scenarios. Figure 8 shows 
Table 1 - Time consumption and leg detection performance of different weak classifiers

\begin{tabular}{||c|c|c|c||}
\hline Item & Linear SVM & WLDA with fixed threshold & WLDA with Gentle Adaboost \\
\hline \hline Detection time/s & 80.22 & 0.1982 & 0.2257 \\
\hline Training time/s & 27600 & 8979.1 & 9807.9 \\
\hline Detection rate/\% & 92.64 & 92.23 & 93.32 \\
\hline
\end{tabular}

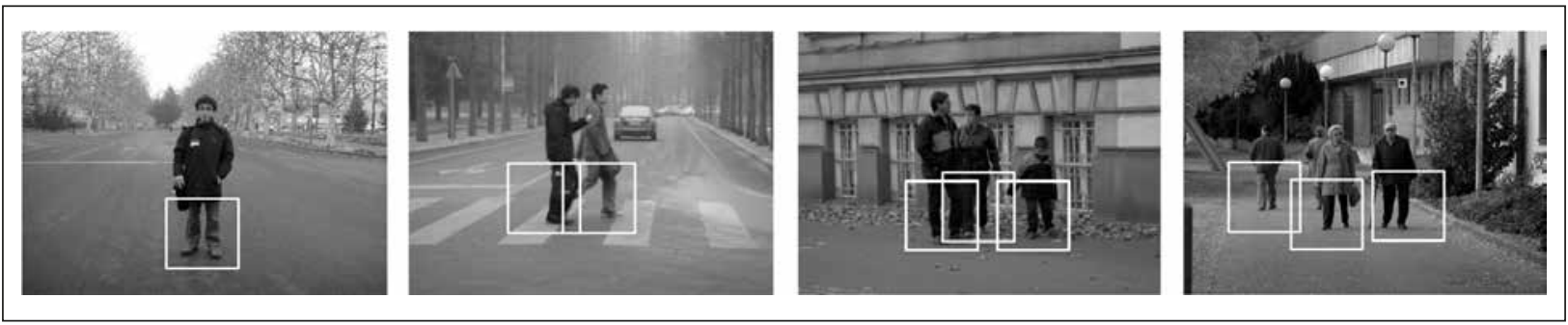

Figure 7 - Example of pedestrian leg detection

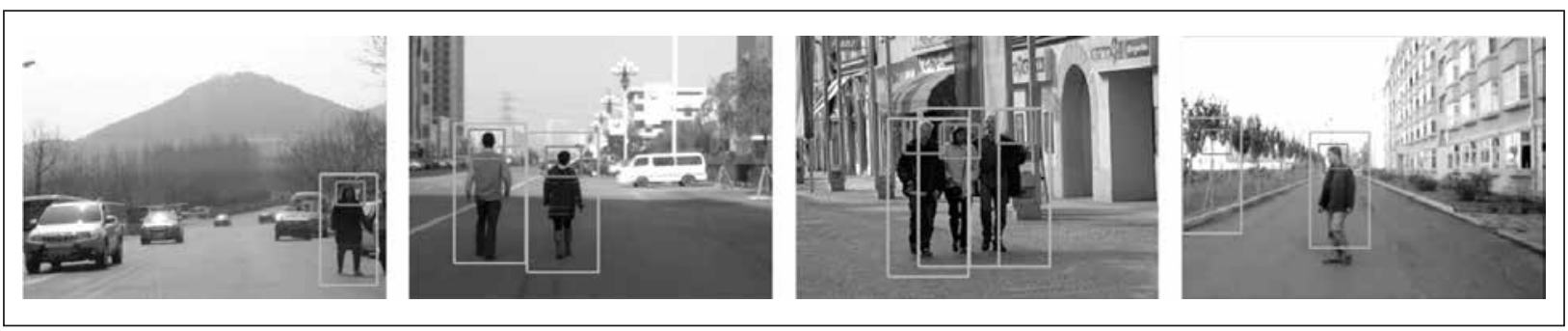

Figure 8 - Pedestrian detection results in real traffic scenario

Table 2 - Body parts-based pedestrian detection results on testing samples

\begin{tabular}{||c|c|c||}
\hline Item & Pedestrian & $\begin{array}{c}\text { Non- } \\
\text { pedestrian }\end{array}$ \\
\hline \hline Number of samples & 890 & 906 \\
\hline Accurately detected number & 820 & 861 \\
\hline Detection rate/\% & 92.1 & 95.0 \\
\hline
\end{tabular}

the final pedestrian detecting results in different environments.

As can be seen from the results, the proposed detection method can realize the single pedestrian, as well as multi-pedestrian, in different real traffic environments. Still, there are some false detections because of the complex background and the pedestrian-like disturbances, as shown in the last image of Figure 8. Usually, the false detection target may not appear and the detected pedestrian may not disappear in front of the vehicle suddenly; such false alarms can be avoided by tracking the detected pedestrian in real urban traffic circumstances.

\section{CONCLUSION AND FUTURE WORK}

Pedestrians are the most important and vulnerable traffic participants in urban transportation system. The necessity to protect them has aroused much attention worldwide, both in the field of automotive safe- ty and advanced driver assistance system. This paper presents a pedestrian detection method based on combined feature of body parts. Different features are used to detect pedestrian's different parts according to its characteristics. Unlike the traditional HOG-based pedestrian method utilizing linear SVM to train the samples, the WLDA is adopted to reduce the dimension of HOG features concentrated on the half part of the sample. The conventional threshold weak classifiers are replaced by LUT weak classifiers. Then parts constraint and classifier threshold adjustment are adopted to fuse the detected key body parts. Parts constraints help to reduce the scope of template matching as well as improve the matching precision and instantaneity. Experimental results verify the effectiveness of the proposed pedestrian detection method.

Currently, the application of vision-based automobile safety system has some key technologies to figure out, such as the reliability and robustness to different weather and illumination conditions. This work in this paper still has some shortages. The main challenge came out during the research of the precise location of pedestrian head which is due to the complex surrounding or disturbances. The key to realize head detection is to gain a region with high discriminative features. Further research should be done to extract more reliable and stable results to enhance the head detection precision. Moreover, pedestrian detection is the previous work of active pedestrian protection. How 
to analyse the hazardous status of the pedestrian according to the pedestrian trajectory still needs to be concentrated on.

\section{ACKNOWLEDGEMENT}

This work was supported by the National Natural Science Foundation of China under Grant Number 51575079 and 51305065.

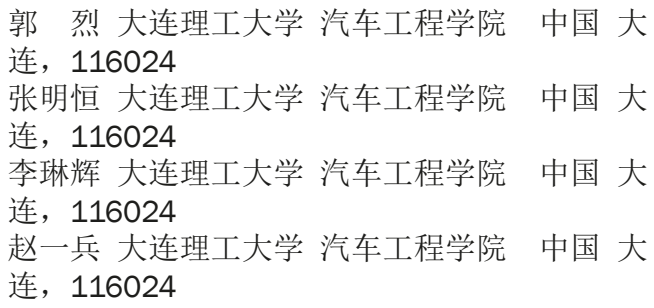

Yingzi Lin Department of Mechanical and Industrial Engineering, College of Engineering, Northeastern University, Boston, MA02115, USA

摘要:

面向行人主动安全的基于身体特征的行人识别方法

为了帮助驾驶员在市区环境下更好地感知前方运动的 行人，提出一种基于行人身体各部位特征的行人识别方 法。根据行人的头部、躯干以及腿部在图像中所呈现特征 的不同，将行人进行分块检测，从而提高复杂交通环境下 行人识别算法的准确性和实时性。根据行人腿部区域的梯 度直方图特征, 利用Gentle Adaboost算法训练得到分割行 人腿部区域的分类器。根据行人头肩模型的边缘特征, 利 用模板匹配方法在腿部区域上方特定区域确定行人头部的 位置。通过人体各部位位置特征约束和调整决策阈值来融 合行人腿部和头部检测结果, 判断是否存在行人。实际市 区环境试验验证了本文算法的可靠性, 结果表明算法能够 达到92.1\%的行人检测率，平均耗时0.2257秒。

关键词。

汽车安全; 行人保护; Gentle AdaBoost; 模板匹配

\section{REFERENCES}

[1] World Health Organization. Global status report on road safety 2013: supporting a decade of action. 2013, Geneva, Switzerland.

[2] NHTSA. Quick Facts 2012. USA: N HTSA's National Center for Statistics and Analysis, Publication Number DOT HS 812 006, 2013.

[3] Zhang XJ, Yao H, Hu GQ, Cui MJ, Gu Y, Xiang HY. Basic characteristics of road traffic deaths in China. Iranian Journal of Public Health. 2013;42(1):7-15.

[4] Traffic Management Bureau of the Ministry of Public Security. 2010 Annual Statistical Report on The People's Republic of China Road Traffic Accidents. China, 2011.

[5] Zegeer CV, Bushell M. Pedestrian crash trends and potential countermeasures from around the world. Accident Analysis \& Prevention. 2012;44(1):3-11.

[6] Hamid HH. The NHTSA's evaluation of automobile safety systems: Active or passive? Loyola Consumer Law Review. 2007;19(3):227-255.
[7] Šimunović L, Bošnjak I, Mandžuka S. Intelligent transport systems and pedestrian traffic. Promet - Traffic \& Transportation. 2009;21(2):141-152.

[8] Kopczynski A, Ptak M, Harnatkiewicz P. The influence of frontal protection system design on pedestrian passive safety. Archives of Civil and Mechanical Engineering. 2011;11(2):345-364.

[9] Matsui Y, Han Y, Mizuno K. Performance of collision damage mitigation braking systems and their effects on human injury in the event of car-to-pedestrian accidents. Stapp Car Crash Journal. 2011;55(4):461-478.

[10] Hu JW, Klinich KD. Toward designing pedestrian-friendly vehicles. Report No. UMTRI-2012-19, University of Michigan, 2012

[11] Broggi A, Cerri P, Ghidoni S, Grisleri P, Jung HG. Active pedestrian protection system, scenario-driven search method for. In: Ehsani M, Wang FY, Brosch GL, editors. Transportation Technologies for Sustainability. Springer Reference; 2013. p. 74-99.

[12] Rosén E, Kallhammer JE, Erikssona D, Nentwich M, Fredriksson R, Smith K. Pedestrian injury mitigation by autonomous braking. Accident Analysis \& Prevention. 2011;42(6):1949-1957.

[13] Keller CG, Thao D, Fritz H, Joos A, Rabe C, Gavrila DM. Active pedestrian safety by automatic braking and evasive steering. IEEE Transactions on Intelligent Transportation Systems. 2011;12(4):1292-1304.

[14] Viola P, Jones M. Rapid object detection using a boosted cascade of simple features. In: IEEE Conference On Computer Vision And Pattern Recognition; 2001.

[15] Lowe DG. Distinctive image features from scale-invariant keypoints. International Journal of Computer Vision. 2004;60(2):91-110.

[16] Dalal N, Triggs B. Histograms of oriented gradients for human detection. In: IEEE Conference on Computer Vision and Pattern Recognition; San Diego, CA, USA; 2005. p. 886-893.

[17] Lu WL, Little JJ. Simultaneous tracking and action recognition using the PCA-HOG descriptor. In: the 3rd Canadian Conference on Computer and Robot Vision; Jun 2006. p. 6-12.

[18] Gan GL, Cheng J. Pedestrian detection based on HOGLBP Feature. In: International Conference on Computational Intelligence and Security; Hainan, China; Dec 2011. p. 1184-1187.

[19] Armanfard N, Komeili M. TED: A texture-edge descriptor for pedestrian detection in video sequences. Pattern Recognition. 2012;45(3):983-992.

[20] Zhang L, Wu B, Nevatia R. Pedestrian detection in infrared images based on local shape features. In: IEEE Conference Computer Vision and Pattern Recognition; Minneapolis, Minnesota, USA; 2007.

[21] Sun RW, Rui T, Zhang JL, Zhou Y. Pedestrian detection by PCA-based mixed HOG-LBP features. In: Proceedings of the 4th International Conference on Internet Multimedia Computing and Service; Wuhan, China; 2012. p. 92-95.

[22] Prioletti A, Mogelmose A, Grisleri P, Trivedi MM, Broggi A, Moeslund TB. Part-based pedestrian detection and feature-based tracking for driver assistance: real-time, robust algorithms, and evaluation. IEEE Transactions on Intelligent Transportation Systems. 2013;14(3):1346-1359. 
[23] Guo L, Ge PS, Zhao YB, Zhang MH, Li LH. Pedestrian detection based on HOG features optimized by gentle AdaBoost in ROI. Journal of Convergence Information Technology. 2013;8(2):554-562.

[24] Laptev I. Improvements of object detection using boosted histograms. Journal of Image and Vision Computing. 2009;27(5):535-544.

[25] Negri P, Clady X, Hanif SM, Prevost L, A cascade of boosted generative and discriminative classifiers for vehicle detection. EURASIP Journal on Advances in Signal Processing. 2008;2008:1-12.

[26] Wu BH, Ai Z, Huang C. LUT-based AdaBoost for gender classification. In: International Conference on Audioand Video-Based Biometric Person Authentication; Guildford, UK; Jun 2003. p. 104-110.

[27] Gavrila DM, Munder S. Multi-cue pedestrian detection and tracking from a moving vehicle. International Journal of Computer Vision. 2007;73(1):41-59.

[28] Gavrila DM. Pedestrian detection from a moving vehicle. Lecture Notes in Computer Science. 2000;1843:37-49.

[29] Katz I, Aghajan H. Multiple camera-based chamfer matching for pedestrian detection. In: IEEE Interna- tional Conference on Distributed Smart Cameras; Stanford, CA, USA; Sep 2008. p. 1-5.

[30] Wu B, Nevatia R. Detection and tracking of multiple, partially occluded humans by Bayesian combination of Edgelet based part detectors. International Journal of Computer Vision. 2007;75(2):247-266.

[31] Yang Y, Ren MW, Yang JY. Obstacles and pedestrian detection on a moving vehicle. International Journal of Advanced Robotic Systems. 2014;11:53. doi: 10.5772/58315.

[32] Pérez Grassi A, Frolov V, Puente León F. Information fusion to detect and classify pedestrians using invariant features. Information Fusion. 2011;12(4):284-92.

[33] Wang C-CR, Wu J-Y, Lien J-JJ. Pedestrian detection system using cascaded boosting with invariance of oriented gradients. International Journal of Pattern Recognition and Artificial Intelligence. 2009;123(4):801-823.

[34] Guo L, Ge PS, Zhang MH, Li LH, Zhao YB. Pedestrian detection for intelligent transportation systems combining AdaBoost algorithm and support vector machine. Expert Systems with Applications. 2012;39(4):42744286. 